\title{
Corona-Kollateralschäden: Informationen, Demonstrationen und Operationen
}

\author{
Korrespondenzadresse \\ Prof. Dr. Dr. Manfred Spitzer \\ Universität Ulm \\ Leimgrubenweg 12-14 \\ 87054 Ulm
}

\author{
Bibliografie \\ DOI https://doi.org/10.1055/a-1134-8996 \\ Nervenheilkunde 2020; 39: 444-451 \\ (c) Georg Thieme Verlag KG Stuttgart · New York \\ ISSN 0722-1541
}

Nach mehr als 4 Monaten Pandemie ist weiterhin kein Ende in Sicht. Im Gegenteil: Weltweit nimmt das Infektionsgeschehen etwa seit Mitte Mai wieder an Fahrt auf, nachdem es im April einen Übergang vom exponentiellen in ein nur noch lineares Wachstum gegeben hatte ( $\triangleright$ Abb. 1). Dieser Hoffnungsschimmer ist mit weltweit 150000 Neuinfektionen pro Tag (erstmals am Donnerstag 18.6.2020) [8] vorüber. Das Zentrum der Pandemie ist mittlerweile vom Osten Chinas über Europa hinweg zum amerikanischen Kontinent gewandert, wo das Virus SARS-CoV-2 aufgrund vielfacher Fehleinschätzungen zweier völlig unfähiger, krankheitsuneinsichtiger, populistisch-konservativer Machthaber, im Norden Trump und im Süden Bolsonaro, zu erschreckenden Zahlen von Infizierten und Toten geführt hat. Das ist kein Zufall, denn das Virus deckt gnadenlos die Schwächen von Gesellschaftssystemen auf, in denen die Menschen unter Bedingungen leben müssen, die nicht nur menschenunwürdig sind, sondern tödlich, sobald eine Zusatzbelastung eintritt.

Mit mehr als 2,5 Millionen Infizierten und mehr als 125000 Todesfällen insgesamt sind die USA seit langer Zeit trauriger Spitzenreiter beim pandemischen Geschehen - Tendenz steigend. Am 28.6.2020 lag die Zahl der Neuinfektionen in den USA bei 44782 , und damit am fünften Tag in Folge jeweils höher als am Vortag [12]. Die Lage dort je nach Ort sehr unterschiedlich ( $\triangleright$ Abb. 2): Städte wie New York scheinen das Schlimmste hinter sich zu haben, befürchten jedoch eine „zweite Welle“. In Florida, Texas, Arizona, New Mexiko, Kalifornien, Utah und Oregon wird die Aufhebung des Lockdowns verschoben oder der Lockdown teilweise wieder eingeführt. So wurden beispielsweise Ende Juni die Bars in Texas und Florida wenige Wochen nach ihrer Wiedereröffnung erneut geschlossen. In Texas nahmen die Corona-bedingten stationären Aufnahmen über die letzten 16 Tage täglich zu, mit 5523 stationär behandelten Covid-19-Fällen am 28.6.2020. Die Intensivstationen des Texas Medical Center waren zu diesem Zeitpunkt zu $100 \%$ ausgelastet [22, 28]. In vielen eher ländlichen Regionen stieg erst Anfang Juni die Zahl der Infektionsfälle: Alaska, Arizona, Arkansas, Kalifornien, Florida, Kentucky, New Mexico, North Carolina, Mississippi, Oregon, South Carolina, Tennessee, Texas und Utah [17]. Dort hat man also den Höhepunkt der „ersten Welle“ noch vor sich.

Die erste Pandemie des 21. Jahrhundert wird von uns nach wie vor hauptsächlich mit Maßnahmen aus dem 14. Jahrhundert bekämpft: Hände waschen und Distanz halten. Aber es gibt Hoff- nung: Das Blutplasma von zuvor an Covid-19 erkrankten Menschen kann schwer Erkrankten helfen [4, 15], der Ausgleich von Vitamin-D-Mangel wahrscheinlich auch [32]. Für einige Medikamente - Blutverdünner (Heparin [20]), Virostatika (Remdesivir [2, 7] und Entzündungshemmer (Dexamethason [13]) - liegen mittlerweile positive Erfahrungen sowie neue Erkenntnisse zum Wirkungsmechanismus vor: Das Virus verursacht nicht nur eine schwere Infektion der Lunge, sondern auch eine überschießende Blutgerinnung und Entzündungsreaktion, auch an den Innenwänden von Blutgefäßen (Endothelialitis [1, 26]). Meinte man noch im April, dass Corona-positive Patienten, die einen schweren Herzinfarkt oder Schlaganfall erleiden, nicht an Corona, sondern „nur“ mit Corona verstorben sind, so wissen wir mittlerweile, dass auch Menschen mittleren Alters (in ihren 30ern und 40ern) davon betroffen sein können - als Folge von Corona. Das Durchschnittsalter von Schlaganfallpatienten liegt „normalerweise“ bei 76 Jahren [19]. Weiterhin wurde zwischenzeitlich deutlich, dass das Virus neben der Lunge auch das Herz, die Nieren, das Gehirn, den Darm (auch das Microbiom) und die Bauchspeicheldrüse (Diabetes!) betreffen kann, samt diese Organe betreffende chronische Syndrome [10, 33].

Wir kennen das Virus SARS-CoV-2 also immer besser und können die von ihm verursachte Krankheit Covid-19 immer besser behandeln. Zudem hat sich gezeigt, dass man die Corona-Krise in keinem Land der Welt mit besseren Chancen zugebracht haben konnte als in Deutschland, vielleicht abgesehen von Neuseeland: Nimmt man verschiedenen Indizes für ein gutes oder schlechtes Abschneiden (Funktion des Gesundheitssystems, Anzahl der durchgeführten Tests, Anzahl der überzähligen Todesfälle, etc.) zusammen, dann landet Deutschland im Vergleich mit 20 anderen Ländern der OECD auf Platz 2 ( $>$ Abb. 3) [27]. Nach einer im Fachblatt Nature publizierten Studie zeigen entsprechende Modellierungen, dass die Maßnahmen des Lockdowns uns bis zum 4.5.2020 in Deutschland allein 530000 Todesfälle, und europaweit 3100000 Todesfälle, erspart haben [9].

Zugleich wird jedoch ebenfalls deutlich, dass unser Umgang mit der Pandemie Kollateralschäden zur Folge hatte, die durch die Pandemie und unsere Reaktionen darauf, nicht aber direkt durch das Virus, verursacht wurden - von sinnlosen vor allem über das Internet verbreiteten Verschwörungstheorien bis zu den sinnvollen Maßnahmen zur Bekämpfung der Pandemie -, die uns noch 


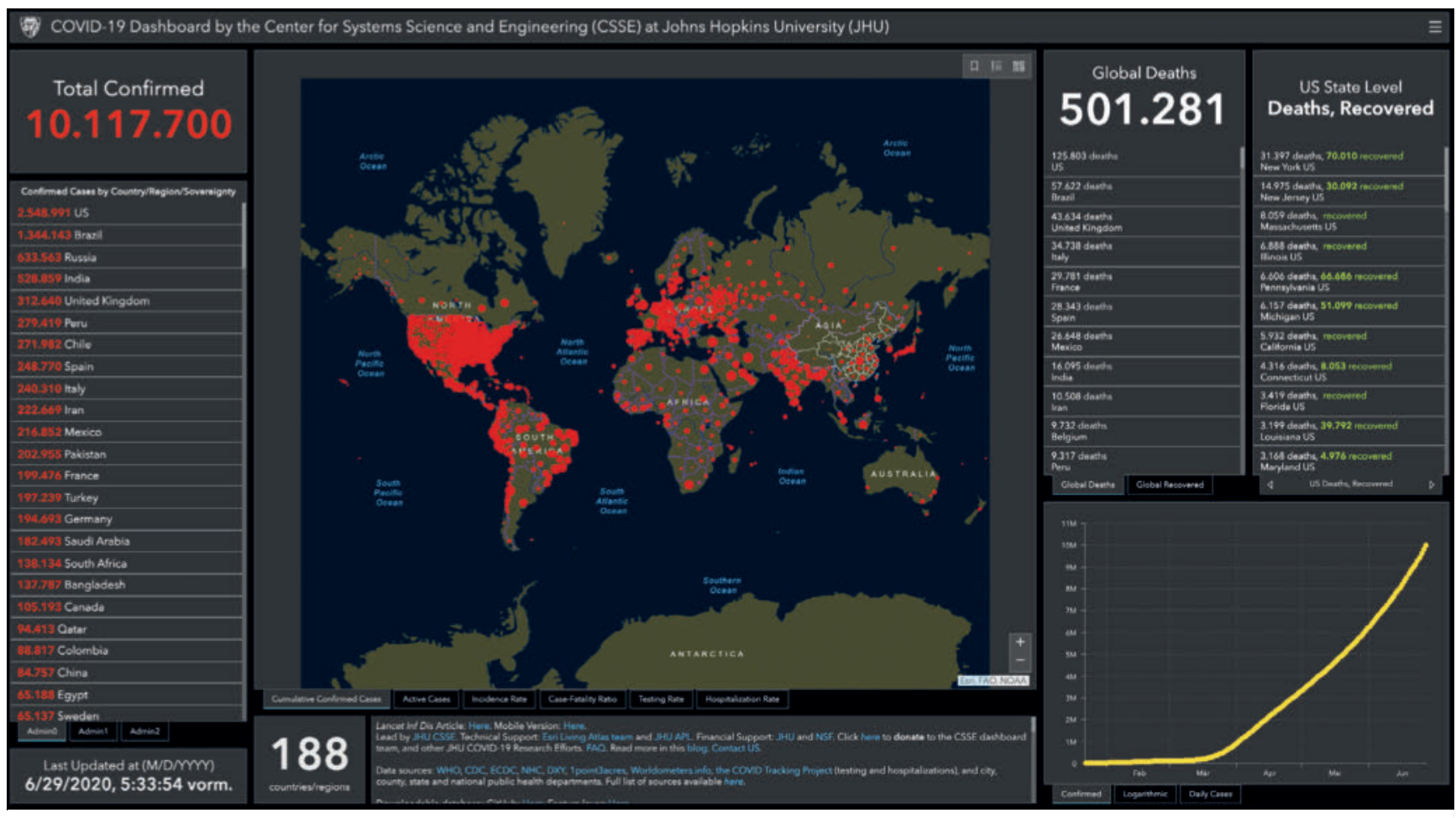

- Abb.1 Screenshot des Dashboard der Johns-Hopkins-University vom 29.6.2020. Mit mehr als 10 Millionen Infizierten und mehr als einer halben Millionen Toten nimmt die Pandemie weiter ungebrochen und mittlerweile wieder beschleunigend ihren Lauf, wie die Kurve der weltweiten Neuinfektionen über die Zeit hinweg rechts unten zeigt. Ein Vergleich zu früheren Dashboards vom 6.4.2020 [23] oder vom 18.5.2020 [24] lohnt sich und zeigt die Dramatik der Situation - gerade jetzt, wo hierzulande alle denken, es sei im Grunde vorbei (Quelle: https://coronavirus.jhu. edu/).

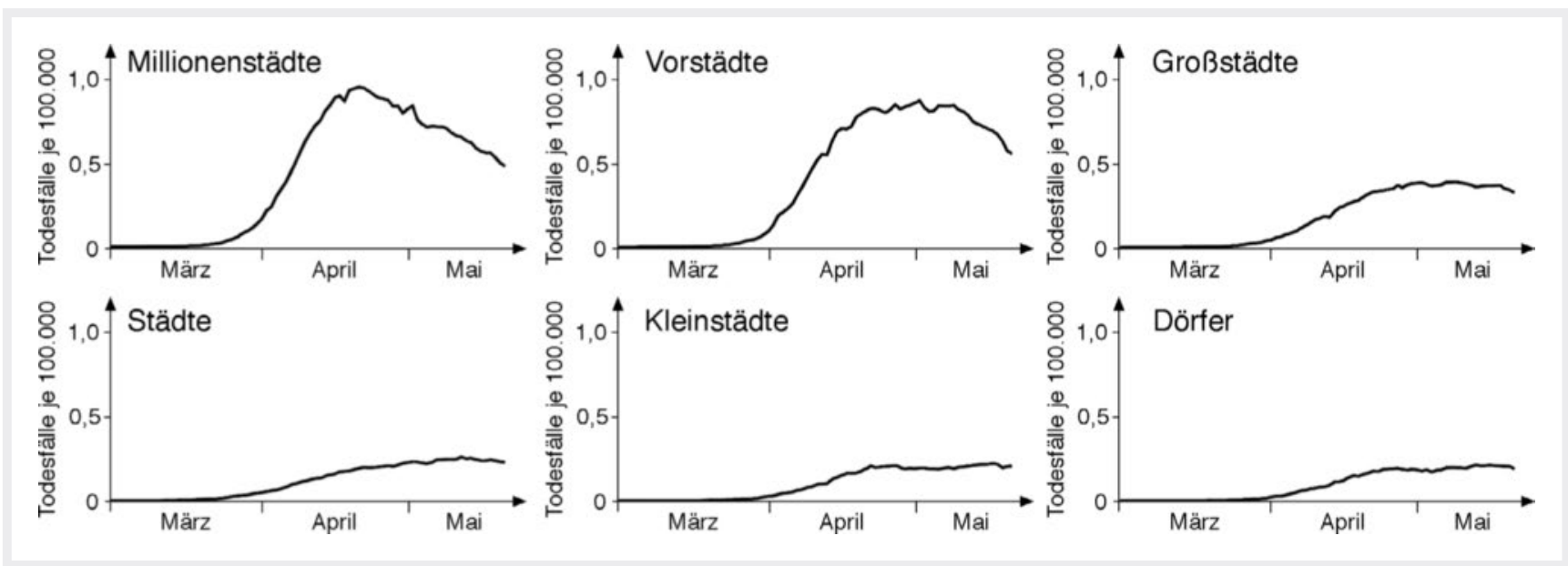

Abb. 2 Verlauf der Covid-19-Todesfälle in verschiedenen Regionen der USA (14-Tage-Durchschnitt) (nach Daten aus [28]). In den großen Städten geht es abwärts, auf dem Lande hingegen aufwärts, wobei der Trend im Juni noch wesentlich deutlicher wurde.

sehr lange betreffen und wissenschaftlich beschäftigen werden. Ein Beispiel aus der Vergangenheit mag dies verdeutlichen: Der Ebola-Ausbruch in Westafrika in den Jahren 2014-2015 hatte nach heutigem Stand 11316 Todesfälle zur Folge. Hinzu kommen jedoch weitere 10623 Todesfälle durch andere Erkrankungen (vor allem Tuberkulose, Malaria und HIV-AIDS), die aufgrund einer etwa 50 \%igen Reduktion des Zugangs zu medizinischen Ressourcen weniger effektiv behandelt werden konnten. Anhand der vorhande- nen Daten aus den damals hauptsächlich betroffenen westafrikanischen Ländern wurde geschätzt, dass es in Sierra Leone 2819 (Cl: 8,44-48,44), in Liberia 1535 (Cl: 5,22-28,78) und in Guinea 6269 (CI: 2,564-12,407) zusätzliche Todesfälle durch die 3 Erkrankungen gegeben hat [21]. Damit verursachte die Ebola-Epidemie nahezu ebenso viele indirekte Todesfälle wie direkte Tote durch das Ebola-Virus. Weltweit sind vom Coronavirus mehr als 10 Millionen Menschen betroffen. Von der Pandemie hingegen sind nahezu alle 


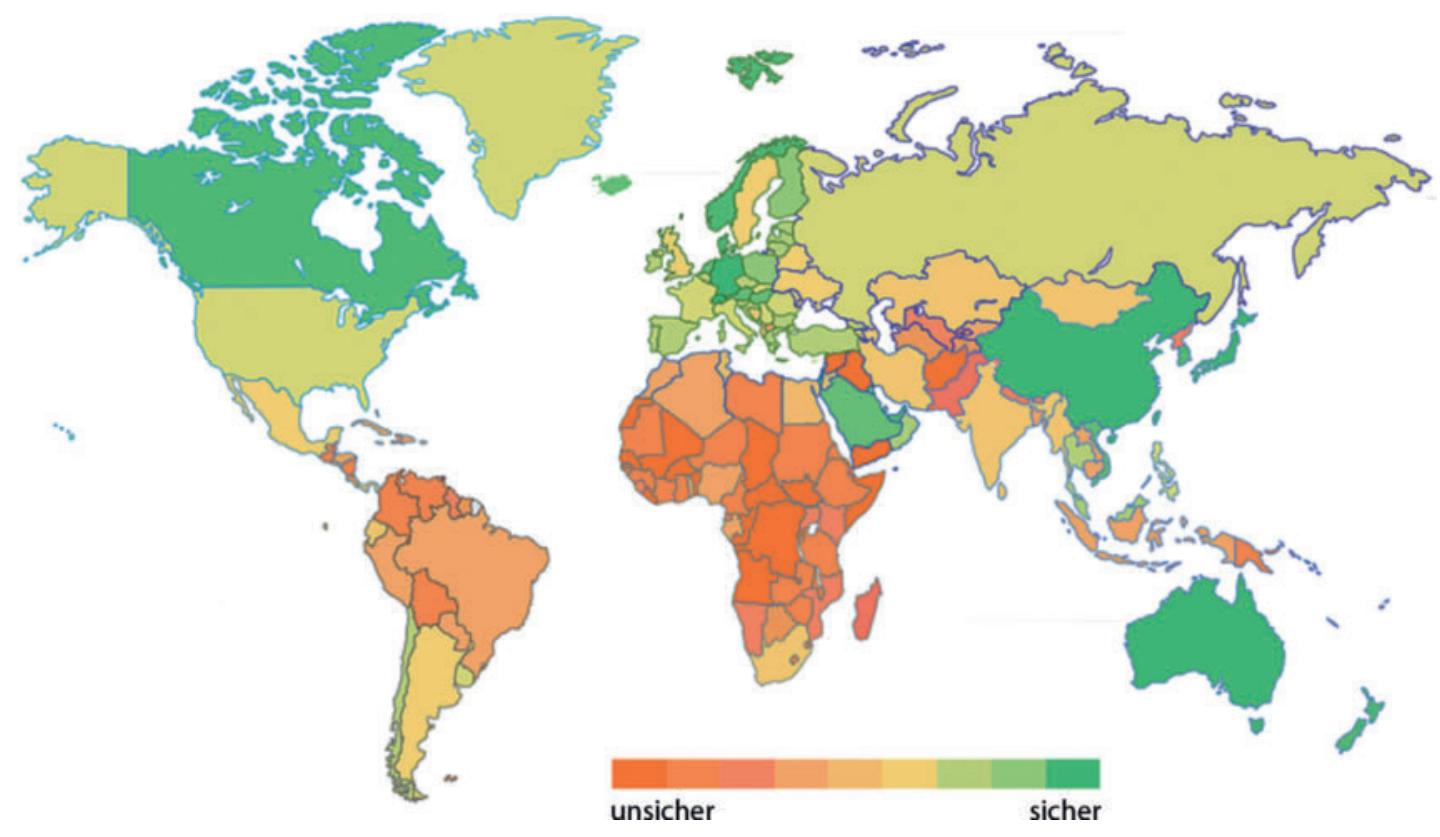

-Abb.3 Globales Ausmaß des Risikos durch Covid-19 im Vergleich von 200 Ländern. Deutschland liegt sehr gut im grünen Bereich (nach Daten aus [5]).
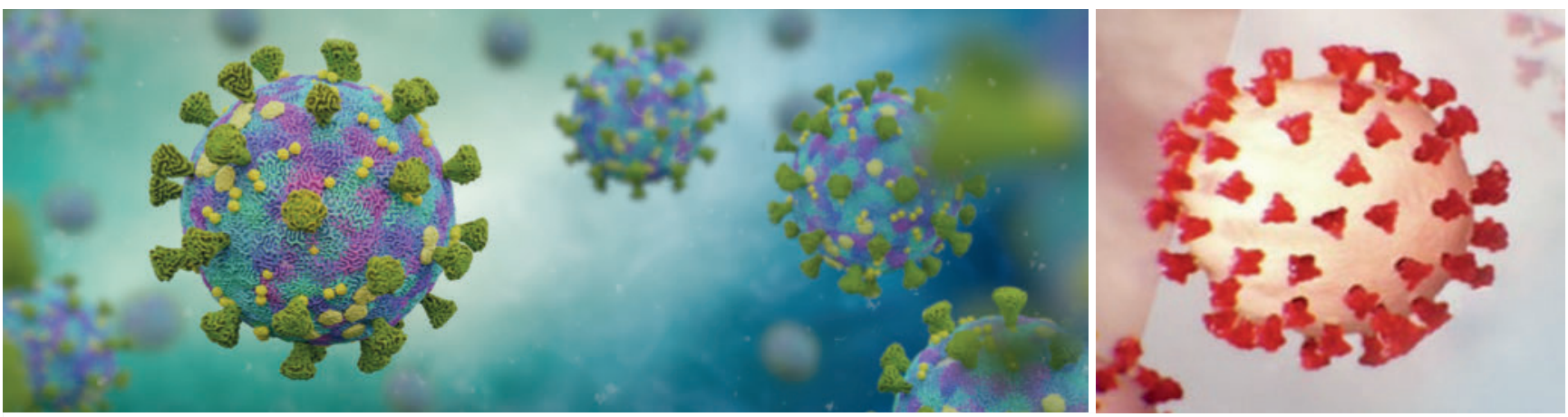

- Abb. 4 Möge sich das wahre Coronavirus doch bitte zu erkennen geben. Die bunten Bilder des Coronavirus sind Produkte der Fantasie von Grafikern. Alles Fake! (Quellen: links: @Adobe Stock/dottedyeti; rechts: Tagesschau)

Menschen in irgendeiner Form betroffen oder werden noch betroffen sein. ${ }^{1}$ Vom Lockdown in der Pandemie waren immerhin etwa 2,6 Milliarden Menschen, also 260-mal mehr als Coronainfizierte Menschen, betroffen.

\section{Infodemie}

Der erste von der Weltgesundheitsorganisation (WHO) bereits im Februar 2020 beklagte Kollateralschaden wurde von ihr selbst als Infodemie bezeichnet. „Wir bekämpfen derzeit nicht nur eine Epidemie, sondern auch eine Infodemie“, sagte der Generalsekretär der WHO, Tedros Adhanom Ghebreyesus, am 15.2.2020 auf der Münchner Sicherheitskonferenz. Damit meinte er die vielen Ge-

1 So geht man beispielsweise von einer kommenden Nahrungsmittelknappheit aus, die möglicherweise die während der Finanzkrise vor 12 Jahren zu verzeichnende Knappheit noch übertreffen könnte [30]. rüchte und Falschmeldungen, die sich über soziale Online-Medien wie Facebook oder Twitter bekanntermaßen sehr rasch verbreiten, nicht zuletzt gerade deshalb, weil sie falsch und damit interessanter sind als wahre Nachrichten [31]. Im Bereich der Medizin kann Falschheit jedoch tödlich sein, weswegen die WHO mehrfach die Geschwindigkeit und die Folgen der Verbreitung von Fake-News über das neue Coronavirus mit der Verbreitung des Virus selbst verglich. Mit dem Ergebnis: Fake-News sind noch schneller! Auch die ganz normalen Nachrichten sind von der Infodemie betroffen. Bunte Bilder von Coronaviren ( $>$ Abb. 4) sehen wir überall, zum Beispiel auch in der Tagesschau ( $\triangleright \mathbf{A b b}$. $\mathbf{5}$, unten), und in den seriösesten wissenschaftlichen Fachblättern (Science, Nature etc.) ist von Epizentren der Pandemie die Rede. Beides gibt es aber gar nicht. Wie sich das wahre Coronavirus im Elektronenmikroskop darstellt, zeigt $>$ Abb. $\mathbf{5}$ (oben) und warum weder eine Epidemie noch eine Pandemie ein Epizentrum hat, zeigt $>$ Abb. 6. Von einem „Epizentrum “ ist immer dann die Rede, wenn es um Erdbeben geht, denn 

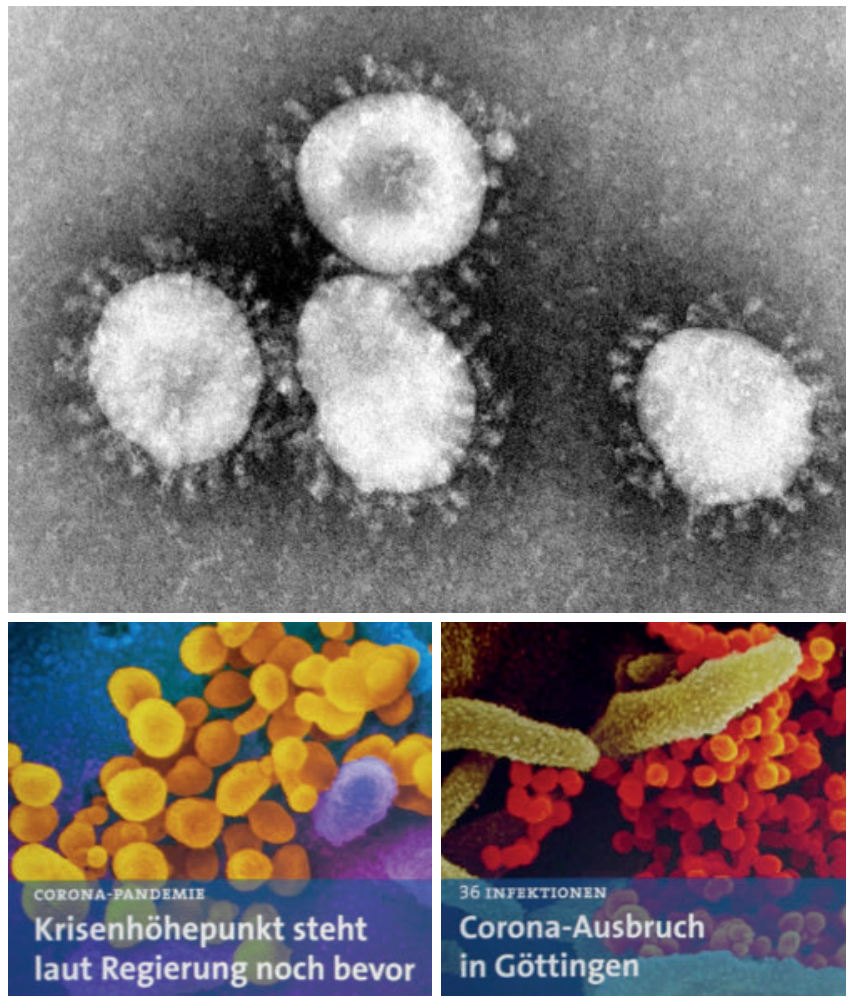

Abb. 5 Aufnahme von Coronaviren mit dem Elektronenmikroskop (oben; Quelle: Hans R. Gelderblom, Freya Kaulbars/RKI). Solche Aufnahmen sind grundsätzlich schwarz-weiß. Oft ist man damit jedoch nicht zufrieden und färbt die Bilder ein, wie z. B. in der Tagesschau (unten). Dabei werden die Farben willkürlich gewählt.

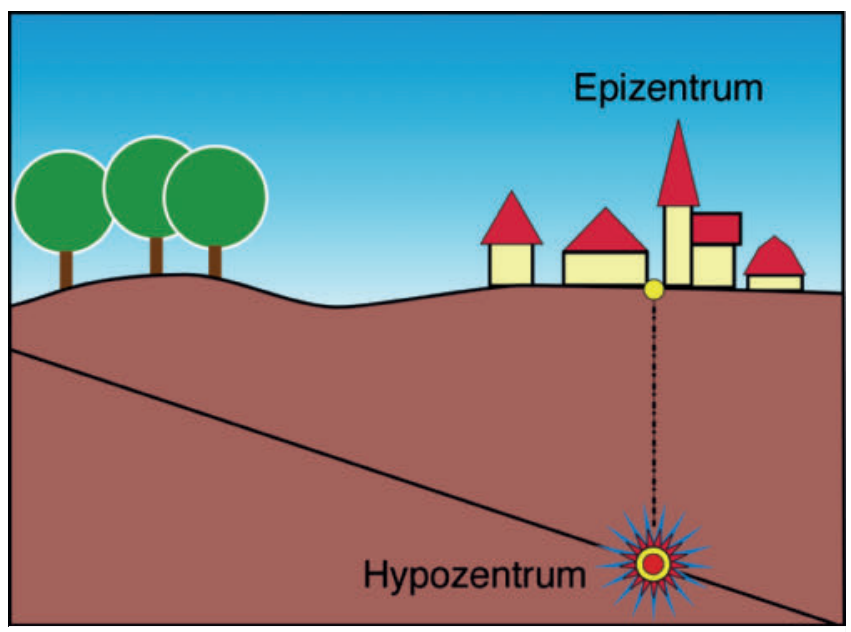

- Abb. 6 Epizentrum und Hypozentrum eines Erdbebens. Epidemien und Pandemien haben ein Zentrum, das die Region oder Stadt bezeichnet, in der es gerade am meisten Neuinfektionen gibt. Ein Epizentrum haben sie nicht (Quelle: nach Wikipedia Commons).

das Zentrum eines Erdbebens liegt stets einige Kilometer bis einige Hundert Kilometer tief unter der Erde entlang einer Bruchkante, an der sich Landmassen gegeneinander bewegen. Das Zentrum eines Erdbebens liegt daher nicht in San Francisco (1906), Christchurch (2011) oder Zagreb (2020), sondern irgendwo tief darun- ter. Man nennt das Zentrum eines Erdbebens daher auch Hypozentrum (vom griechischen Wort „hypo“ = „darunter“). Die genannten Städte lagen über dem Zentrum der nach ihnen benannten Erbeben, weswegen man mit ihren Namen (und noch genauer dem Längen- und Breitengrad eines Orts an der Erdoberfläche über einem Erdbeben) den Ort eines Erdbebens jeweils genau senkrecht über dessen Mittelpunkt als Epizentrum bezeichnet. „Epi“ kommt aus dem Griechischen und bedeutet „darüber“. Halten wir fest: Das Hypozentrum ist das Zentrum des Erdbebens und liegt unter der Erde. Das Epizentrum des Erdbebens liegt über dem Erdbeben.

Ein Beispiel: New York war im April 2020 weltweit die Stadt mit den meisten Neuinfektionen im Rahmen der Corona-Pandemie, also deren Zentrum. Aber nur wenn sich alle erkrankten New Yorker zu irgendeinem Zeitpunkt einmal beispielsweise in der New Yorker U-Bahn befunden hätten, wäre es zu diesem Zeitpunkt richtig gewesen, New York als Epizentrum der Pandemie zu bezeichnen.

Wen wundert es angesichts dieser größtenteils frei erfundenen Bildersprache und der zuweilen unklaren Verbalsprache, dass der Laie Mühe hat, bei einer Nachricht auch in den ganz normalen Nachrichten (Tagesschau) zu entscheiden, ob sie richtig oder falsch ist? - Seit es die Corona-Pandemie gibt, gibt es auch die verschiedensten Verschwörungstheorien ${ }^{2}$ zum Virus und zu seiner Verbreitung: Das Virus wurde hergestellt „in einem Hochsicherheitslabor“, von „den Chinesen“, von „Bill Gates“, „der Pharma-Industrie“ oder „dem Großkapital“. Nach manchen Theorien sei es besonders gefährlich und soll uns schaden. Nach anderen Verschwörungstheorien schadet es uns gar nicht, aber die Abwehrmaßnahmen gegen das Virus sollen uns schaden. Beide Theorien schließen sich gegenseitig aus, was jedoch manche Verschwörungstheoretiker nicht daran hindert, an beide zugleich zu glauben.

Man kennt dieses Phänomen sich widersprechender „Verschwörungstheorien“ (sie kommen fast immer im Plural) schon lange: Die einen glauben, dass Prinzessin Diana vom CIA oder von einem anderen Geheimdienst umgebracht wurde, und die anderen glauben, dass Prinzessin Diana in Wahrheit gar nicht gestorben ist, sondern irgendwo auf der Welt in Ruhe lebt. Nicht nur, dass es beide Verschwörungstheorien gibt, verwundert einen, sondern vor allem die Tatsache, dass manche Menschen an beide glauben, obwohl sich das logisch widerspricht [25].

Man könnte Verschwörungstheorien als Fantasien belächeln und sie nicht weiter beachten, wenn sie nicht gefährlich wären. Impfskeptiker beispielsweise riskieren den Tod ihrer Kinder und anderer Menschen. Eine im Fachblatt JAMA Internal Medicine im Mai 2014 von US-amerikanischen Wissenschaftlern publizierte Studie zeigte, dass etwa die Hälfte der befragten 1351 Erwachsenen mindestens eine von 6 einzeln erfragten medizinischen Verschwörungstheorien für wahr hielt. Verschwörungstheorien sind also häufig. Sie werden durch Angst vor dem Unbekannten geschürt und sind letztlich nur mit der Einsicht in die Wahrheit behandelbar. Zuwei-

2 Das Wort „Verschwörungstheorie“ sei hier verwendet, obwohl es sich mit ihm wie mit „Epizentrum “ verhält: Es ist in sich widersprüchlich und daher sinnlos. Das Wort „Theorie“ kommt aus dem Griechischen und bedeutet soviel wie „Erkenntnis“ oder „Einsicht“ und meint damit ziemlich genau das Gegenteil von „Verschwörung“. Theorien erlauben Vorhersagen (prognostischer Wert), das Stellen neuer Fragen (heuristischer Wert) und werden angewendet, weil sie nützlich sind (praktischer Wert). 

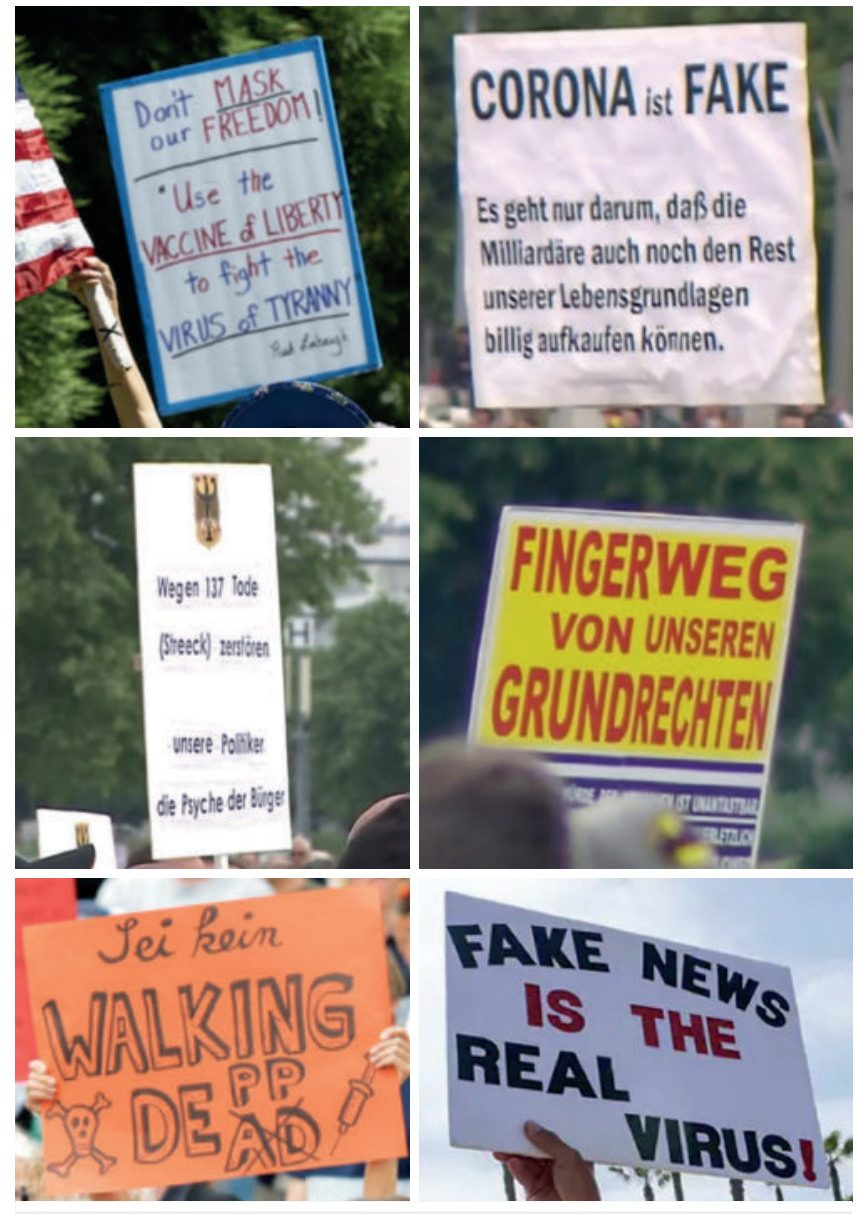

- Abb.7 Plakate, die man hierzulande und anderswo auf Demonstrationen gesehen hat (Quellen: Ausschnitte aus Pressefotos in Print- und digitalen Medien).

len werden sie mit an Wahn grenzender Gewissheit vertreten. Der Psychiater weiß, dass man dann mit Reden nicht viel weiter kommt.

\section{Demonstrationen}

Seit einigen Wochen wird in Deutschland gegen die Maßnahmen des Lockdowns, gegen die Regierung, die diese Maßnahmen implementiert hat und gegen einfach alles demonstriert ( $\mathbf{A} \mathbf{b} \mathbf{b} . \mathbf{7}$ ).

Dass man in Deutschland demonstrieren darf, ist eine große Errungenschaft, und dass man es vor Kurzem für einige Wochen nicht durfte, auch! Die Befürchtungen, Corona werde hierzulande (wie in Brasilien, China, Russland, Tatschikistan, Ungarn oder Weißrussland) dazu verwendet, demokratische Systemkritik zu unterbinden, ist falsch. Unsere Demokratie funktioniert.

Man wundert sich jedoch über manche gemäßigten Demonstranten. Wie eingangs erwähnt, hat Deutschland weltweit die Krise - vielleicht hinter Neuseeland - am besten gemeistert ( $\bullet \mathbf{A b b}$. 8). In Neuseeland ist man stolz auf die Präsidentin. Und was machen wir? Wir demonstrieren gegen die Kanzlerin. Unsere Nachbarn beneiden uns um sie. Die Gründe wird man dank der empirischen Sozialforschung irgendwann erfahren. Aus psychiatrischer Sicht geht es um diffuse Ängste und ins Wahnhafte reichende, durch Fakten offenbar nur schwer zu ändernde Gedanken.

\section{Operationen}

Bei der Corona-Krise geht es um eine Infektionskrankheit. Man denkt daher zunächst nicht daran, dass diese Krise die Chirurgie weltweit in eine Krise gestürzt hat, deren Konsequenzen erst seit Mitte Mai - sehr langsam - ans Tageslicht kommen. Am 8.5.2020 publizierte das Deutsche Ärzteblatt, dass schätzungsweise 1,6 Millionen geplante („elektive“) Operationen im Zeitraum zwischen dem 16.3.2020 und 4.5.2020 aufgrund der Corona-Pandemie verschoben wurden. Man wollte Intensivbetten für Covid-19-Patienten freihalten und hat daher nicht operiert, denn nach einer OP befinden sich viele Patienten auf der Intensivstation. Nur eine Woche später veröffentlichte ein weltweit neu gegründeter Verbund von mehr als 5000 Chirurgen aus mehr als 120 Ländern, der sich CovidSurg Collaborative Gruppe nennt, im Fachblatt British Journal of Surgery vorab als Preprint eine Studie, die uns noch viel zu denken geben, und darüber hinaus noch viel mehr zu arbeiten geben wird: Durch eine beispiellose Sammlung von Daten aus 359 Kliniken in 71 Ländern, die auf 190 Länder hochgerechnet wurden, ermittelte diese Gruppe die Auswirkungen der Corona-Pandemie auf die weltweite chirurgische Praxis. Das Ergebnis: Während der ersten 12 Wochen der Corona-Epidemie wurden weltweit 28 Millionen chirurgische Eingriffe verschoben. In endlosen Tabellen kann man in dieser Arbeit nachsehen, in welchem Land der Welt welche Operationen (und wie viele) nicht erfolgten. Daraus errechnete man dann, dass es mit jeder zusätzlichen Woche der Pandemie weltweit zu etwa 2,4 Millionen weiteren Verschiebungen chirurgischer Eingriffe kommt, wenn man weiterhin Kapazitäten zur Behandlung von Covid-19-Patienten vorhält.

Auch 34 deutsche Kliniken waren an dieser Studie beteiligt, sodass für Deutschland bekannt wurde: 908759 Operationen wurden aufgeschoben, davon etwa 850000 elektive Eingriffe. In Frankreich und den USA wurden 700000 bzw. 3,8 Millionen Operationen verschoben. Aber was heißt schon „elektiv“? Im Grunde nur, dass es sich um keine akuten Notfälle handelte, um die man sich selbstverständlich weiter gekümmert hat. Aber kann man, ja darf man beispielsweise Krebsoperationen, die nicht unbedingt einen akuten Notfall darstellen, aufschieben? - Wenn überhaupt, dann eigentlich nur für die kürzest mögliche Zeit, denn durch diese Eingriffe wird nicht nur Leben gerettet, sondern vor allem auch Leiden vermindert. Die weltweite Betrachtung ergab: Je ärmer ein Land ist, desto häufiger waren die Verschiebungen von Krebsoperationen. Die in Staaten mit ohnehin schon schwachen Gesundheitssystemen lebenden Menschen leiden damit am meisten unter der Pandemie ( $\triangleright$ Abb.9).

Allein für Großbritannien liegt eine Schätzung vor, der zufolge Ende dieses Jahres 10 Millionen OPs aufgeschoben sein werden. Weil dort die Wartezeiten ohnehin schon sehr lange sind (oft Jahre - vor der Corona-Krise!), mag man sich nicht ausmalen, wieviel Leid und Tod die Corona-Pandemie über ihre Auswirkungen auf das Operieren verursacht. Irgendwann wird man es wissen, weil man dieser Frage wissenschaftlich nachgehen kann und dies hoffentlich auch tun wird.

\section{Diskussion}

In wirtschaftlich starken Staaten mit gut funktionierenden Sozialsystemen sollte niemand aufgrund der Pandemie hungern oder 


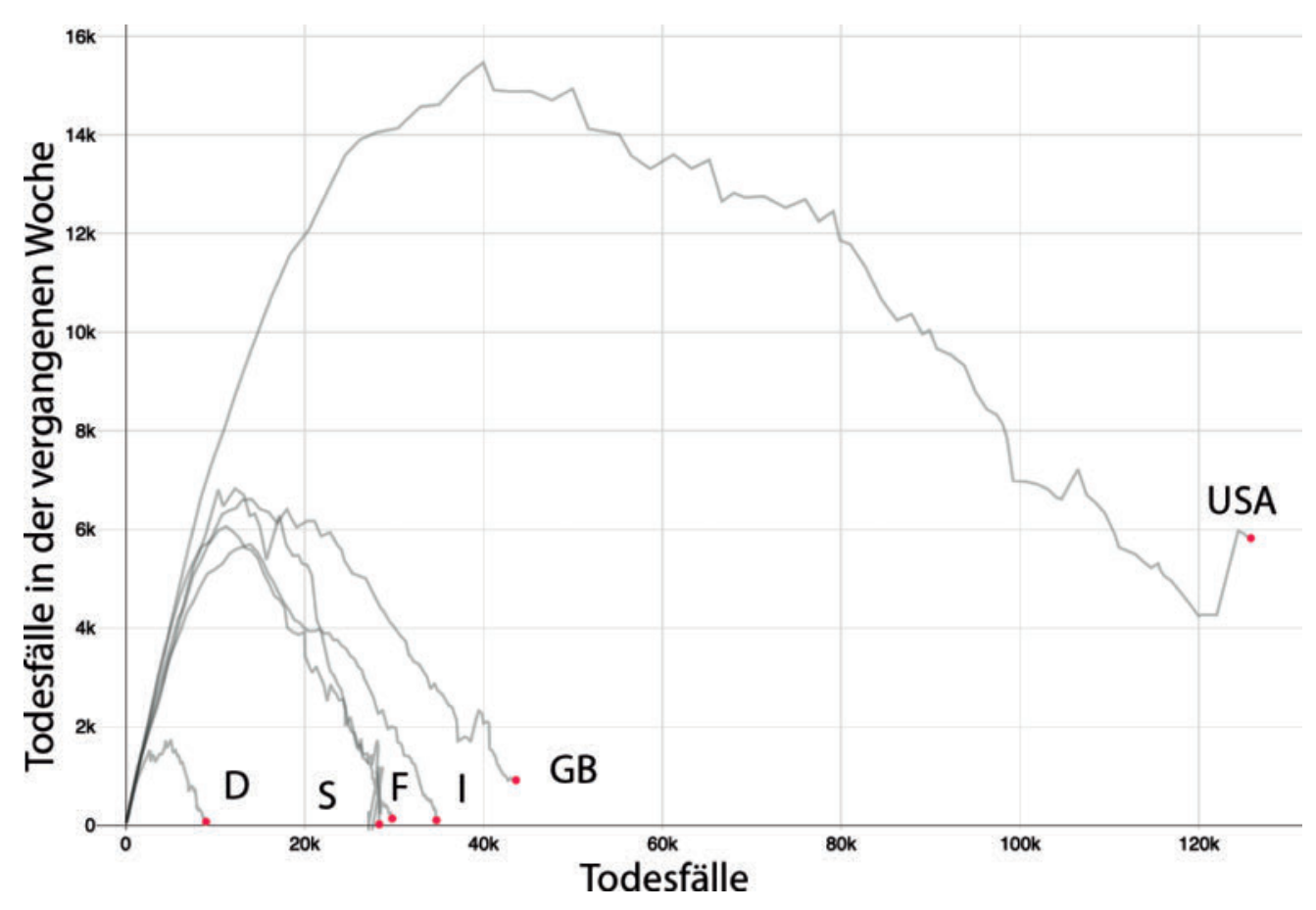

Abb. 8 Todesfälle im Verlauf der Pandemie und deren Dynamik (Todesfälle pro Woche) in Deutschland, Spanien, Frankreich, Italien, Großbritannien und den USA. Bedenkt man nun noch, dass wir nach den USA von den angeführten Ländern die zweitgrößte Bevölkerung haben (ca. 83 Millionen), unser „Berg“ also verglichen mit den „Bergen“ unserer europäischen Nachbarn (bezogen auf die Bevölkerung) vergleichsweise noch kleiner wäre, kann man nur froh sein, während der Pandemie in Deutschland gewesen zu sein (übersetzt und modifiziert nach: https://aatishb.con/covidtrends/ abgerufen am 29.6.2020).

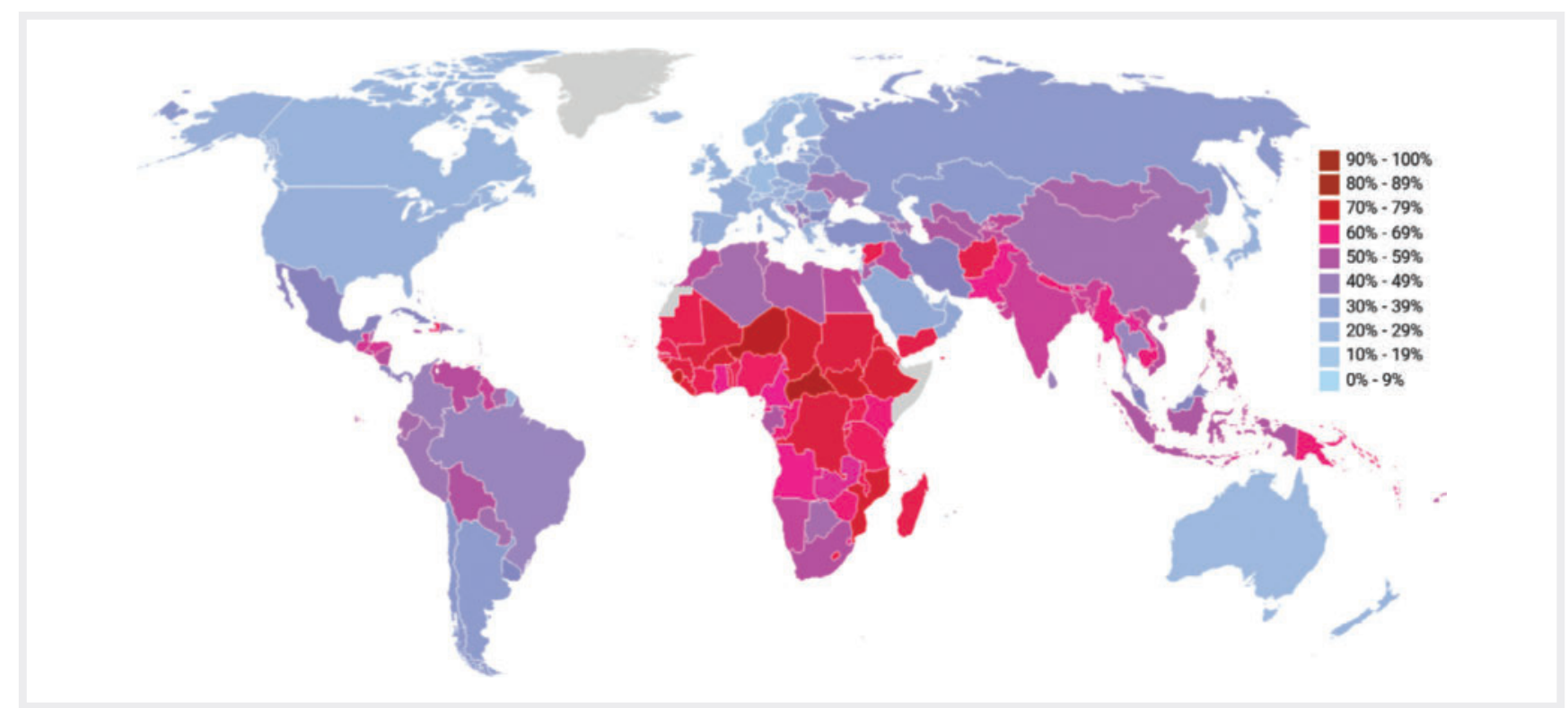

- Abb.9 Abnahme der Krebs-Operationen in Prozent innerhalb von 12 Wochen Corona-Pandemie in verschiedenen Ländern der Erde (je dunkler, desto größer die Abnahme (nach Daten aus [18]).

auf der Straße sitzen müssen. Aber sogar in der Schweiz nimmt die Länge der vor kostenlosen Garküchen oder Nahrungsmittelausgabestellen stehenden Menschenschlangen beängstigend zu. In den USA sowieso. Denn im Land mit der stärksten Weltwirtschaft gibt es nicht nur knapp 30 Millionen Menschen ohne Krankenversicherung, sondern mittlerweile auch über 40 Millionen mehr Arbeitslose als Anfang dieses Jahres. Das befeuert nicht nur die Pandemie (wer nicht krankenversichert ist, geht nicht zum Arzt, und wer hustet, 
wird dennoch arbeiten, um seinen Job nicht zu verlieren), sondern wirkt sich langfristig wahrscheinlich noch gravierender indirekt auf die Gesundheit aus: Existenzieller Stress bei Arbeitslosigkeit, Obdachlosigkeit oder Krankheit schwächt nicht nur die Immunabwehr (und befeuert auch auf diese Weise die Pandemie weiter), sondern führt auch zu sozialem Abstieg und einer Reihe weiterer Krankheiten (Bluthochdruck, Diabetes). Beides, der soziale Abstieg und die Krankheiten erhöhen wiederum die Wahrscheinlichkeit der ohnehin häufigsten Todesursachen in der westlichen Welt (Herzinfarkte und Schlaganfälle) [16]. Wirtschaft und Gesundheit hängen sehr eng zusammen. Für die gegenwärtige Krise gilt, was der ehemalige Vizekanzler Sigmar Gabriel neulich in einem Interview sagte: „Alles, was gesundheitlich schiefgeht, hat hinterher ökonomische Folgen. Alles, was ökonomisch schiefgeht, hat hinterher gesundheitliche Folgen. “ Er fügte hinzu, dass man beides nicht gegeneinander ausspielen könne. Es komme vor allem darauf an, in schwierigen Zeiten die Übersicht und Vernunft zu behalten, um einigermaßen richtig zu entscheiden. Das ist schon sehr viel, und das hat hierzulande vergleichsweise sehr gut funktioniert.

Es liegt an uns, wie wir die Zukunft gestalten und wie die Welt nach der Corona-Krise aussieht. Es darf dabei nicht geschehen, dass wir wegen der Pandemie unsere Werte über Bord werfen. Die Freiheitseinschränkungen wurden weitgehend wieder aufgehoben, begleitet von zusätzlichen Maßnahmen wie Schutzmasken und der Tracking-App, die mit der europäischen Datenschutzgrundverordnung vereinbar ist. In den USA wird deutlich, dass die Menschen dort an einer sehr unglücklichen Kombination schlecht funktionierender Sozial- und Gesundheitssysteme einerseits und eines unfähigen Präsidenten andererseits sterben. Das müsste nicht so sein und könnte sich durch Corona ändern. In einem kürzlich im New England Journal of Medicine erschienen Editorial findet man zu den Auswirkungen von Corona in den USA Folgendes:

„Noch nie zuvor waren die wechselseitigen Abhängigkeiten unserer Gesundheit, unserer Finanzen und unseres sozialen Gefüges so deutlich sichtbar. Und niemals zuvor war die Notwendigkeit von Gesundheitsreformen, die allen Amerikanern den Zugang zu einer erschwinglichen Gesundheitsversorgung sicherstellen, so deutlich erkennbar. Unsere Gesundheitspolitik sollte auf diese Realität reagieren, sowohl während dieser Pandemie als auch in der Zukunft, und wir sollten die Lehren aus dieser Krise nicht tatenlos an uns vorüberziehen lassen “ [14]. ${ }^{3}$ Für uns Europäer gilt, dass wir nachbessern und unsere Gesundheitssysteme nicht mehr ausschließlich nach ökonomischen Gesichtspunkten gestalten sollten. Robustheit und Nachhaltigkeit sollten ebenfalls bedacht werden. Virusausbrüche sind unvermeidlich, Pandemien hingegen lassen sich vermeiden.

Ein Letztes: Das Leben muss nicht in jeder Hinsicht wieder genauso werden, wie es vorher war: Ressourcenverbrauch, Flugreisen, sinnlose Events und vor allem alles immer „schneller, höher, weiter" - wer will das eigentlich noch auf Kosten des Erdballs? Set-

3 „Never before has the interdependence of all our health, finances, and social fabric been so starkly visible. Never before has the need for health care reforms that ensure universal access to affordable care for all Americans been more apparent. Our policies on health and health care, both during this pandemic and in the future, should reflect this reality, and we should not let the lessons of this crisis pass us by." zen wir uns alle dafür ein, dass wir künftig bewusster, nachhaltiger und solidarischer leben.

Literatur

[1] Ackermann M, Verleden SE, Kuehnel M, et al. Pulmonary Vascular Endothelialitis, Thrombosis, and Angiogenesis in Covid-19. N Engl J Med 2020. doi10.1056/NEJMoa2015432

[2] Beigel JH, Tomashek KM, Dodd LE, et al. Remdesivir for the treatment of Covid-19 - preliminary report. N Engl J Med 2020. doi: 10.1056/ NEJMoa2007764

[3] Bellware K, Dupree J. 14 states and Puerto Rico hit highest seven-day average of new coronavirus infections. The Washington Post 9.6.2020 https://www.washingtonpost.com/health/2020/06/08/14-states-puerto-rico-hit-their-highest-seven-day-average-new-covid-19-infectionssince-june/; abgerufen am 10.6.2020

[4] Casadevall A, Joyner MJ, Pirofski LA. A Randomized Trial of Convalescent Plasma for COVID-19 - Potentially Hopeful Signals. JAMA 2020; June 3

[5] Deep Knowledge Group, dkv. COVID-19 Regional Safety Assessment (200 Regions). Safety score of 200 Regions and Territories (Daten vom 3.6.2020) Deep Knowledge Group. https://www.dkv.global/covid-safety-assessment-200-regions; abgerufen am 21.6.2020

[6] Denworth L. The Biggest Psychological Experiment in History Is Running Now. Scientific American, Juni 2020. https://www.scientificamerican.com/interactive/the-biggest-psychological-experiment-in-history-is-running-now/; abgerufen am 26.6.2020

[7] Dolin RC, Hirsch MS. Remdesivir - An important first step. NEJM 2020. doi: 10.1056/NEJMe2018715

[8] Farzan AN, Hassan J, Noack R, et al. Live updates: WHO warns of 'new and dangerous phase' as coronavirus accelerates; Americas now hardest hit. The Washington Post 19.6.2020 online; abgerufen am 19.6.2020

[9] Flaxman, S. et al. Estimating the effects of non-pharmaceutical interventions on COVID-19 in Europe. Nature 2020. doi.org/10.1038/ s41586-020-2405-7

[10] Geddes L. Why strange and debilitating coronavirus symptoms can last for months. New Scientist, 24.6.2020. https://www.newscientist.com/ article/mg24632881-400-why-strange-and-debilitating-coronavirussymptoms-can-last-for-months/; abgerufen am 28.6.2020

[11] Götzinger F, Santiago-García B et al. on behalf of the ptbnet COVID-19 Study Group. COVID-19 in children and adolescents in Europe: a multinational, multicentre cohort study. Lancet Child Adolesc Health 2020. doi.org/10.1016/S2352-4642(20)30177-2; abgerufen am 26.6.2020

[12] Hawkins D, Adam K, Kornfield M, et al. U. S. sets single-day record for new coronavirus cases for fifth consecutive day. The Washington Post, 28.6.2020 https://www.washingtonpost.com/nation/2020/06/27/ coronavirus-live-updates-us/; abgerufen am 28.6.2020

[13] Horby PW, Landray MJ, et al. Effect of Dexamethasone in Hospitalized Patients with COVID-19 - Preliminary Report. medRxiv preprint 2020. doi.org/10.1101/2020.06.22.20137273; abgerufen am 29.6.2020

[14] King JS. Covid-19 and the Need for Health Care Reform. NEJM 2020; 1-3: e104

[15] Li L, Zhang W, Hu Y, et al. Effect of Convalescent Plasma Therapy on Time to Clinical Improvement in Patients With Severe and Life-threatening COVID-19: A Randomized Clinical Trial. JAMA 2020; doi:10.1001/jama.2020.10044

[16] Marmot M. Health in an unequal World. Lancet 2006; 368: 2081-2094

[17] Milman O. Coronavirus: more than a dozen US states see record high of new cases. The Guardian, 9.6.2020. https://www.theguard- 
ian.com/world/2020/jun/09/coronavirus-cases-uptick-detected-some-us-states/; abgerufen am 11.6.2020

[18] Nepogodiev D, Omar OM, Abbott T et al. Elective surgery cancellations due to the COVID-19 pandemic: global predictive modelling to inform surgical recovery plans. Britsish Journal of Surgery 2020. doi. org/10.1002/bjs.11746; abgerufen am 15.5.2020

[19] Oxley T]. Moco J, Majidi S et al. Large-Vessel Stroke as a Presenting Feature of Covid-19 in the Young. NEJM 2020. doi: 10.1056/NEJMc2009787

[20] Paranjpe I, Fuster V, Lala A et al. Association of Treatment Dose Anticoagulation with In-Hospital Survival Among Hospitalized Patients with COVID-19, Journal of the American College of Cardiology 2020. doi. org/10.1016/j.jacc.2020.05.001/; abgerufen am 8.5.2020

[21] Parpia AS, Ndeffo-Mbah ML, Wenzel NS, et al. Effects of Response to 2014-2015 Ebola Outbreak on Deaths from Malaria, HIV/AIDS, and Tuberculosis, West Africa. Emerging Infectious Diseases 2016; 22. doi: 10.3201/eid2203.150977; abgerufen am 3.4.2020

[22] Selk A. Coronavirus Updates. The Washington Post, 27.6.2020 https://s2.washingtonpost.com/camp-rw/?trackld=5c7aa6689bbc0f614961acc4\&s=5ef66434fe1ff6482dad022f\&linknum=1\&linktot=60; abgerufen am 28.6.2020

[23] Spitzer M. Psychologie und Pandemie. Die Auswirkungen des CoronaVirus auf den Einzelnen und auf die Gesellschaft. Nervenheilkunde 2020; 39: 274-283

[24] Spitzer M. Pandemie. Was die Krise mit uns macht und was wir aus ihr machen. München: mvg Verlag, 2020

[25] Spitzer M. Pandemie - historisch, systematisch, kulturell und (sozial-) politisch. Nervenheilkunde 2020; 39: 363-372
[26] Teuwen LA, Geldhof V, Pasut A, et al. COVID-19: the vasculature unleashed. Nat Rev Immunol 2020. doi:10.1038/s41577-020-0343-0

[27] The Economist Intelligence Unit. How well have OECD countries responded to the coronavirus crisis? Coronavirus Whitepaper V3 (Daten vom 9.6.2020) The Economist Intelligence Unit Limited, 17.6.2020 https://www.eiu.com/n/quality-of-oecd-countries-response-to-thepandemic/; bzw. https://www.eiu.com/n/campaigns/oecd-countries-responded-to-the-coronavirus-crisis/; abgerufen am 22.6.2020

[28] Thebault R, Hauslohner A. A deadly ‘checkerboard': Covid-19's new surge across rural America. The Washington Post 24.5.2020. https:// www.washingtonpost.com/nation/2020/05/24/coronavirus-rural-america-outbreaks/?arc404=true\&itid=Ik_inline_manual_9; abgerufen am 27.6.2020

[29] Thebault R, Hauslohner A. Reopenings, record cases and full hospitals: America's dissonant response to the pandemic. The Washington Post, 25.6.2020. https://www.washingtonpost.com/

[30] Vaughan A. Covid-19 pandemic risks worst global food crisis in decades. New Scientist 2020. https://www.newscientist.com/article/2243692-covid-19-pandemic-risks-worst-global-food-crisis-in-decades/; abgerufen am 20.6.2020

[31] Vosoughi S, Roy D, Aral S. The spread of true and false news online. Science 2018; 359: 1146-1151

[32] Whittemore PB. COVID-19 Fatalities, Latitude, Sunlight, and Vitamin D. American Journal of Infection Control 2020. doi.org/10.1016/j. ajic.2020.06.193

[33] Wilson C. The coronavirus is leaving some people with permanent lung damage. New Scientist, 25.6.2020 https://www.newscientist.com/ article/2247086-the-coronavirus-is-leaving-some-people-with-permanent-lung-damage/; abgerufen am 28.6.2020 